\title{
Article \\ Evaluation of ZIF-8 and ZIF-90 as Heat Storage Materials by Using Water, Methanol and Ethanol as Working Fluids
}

\author{
Ciara Byrne $^{1, *(\mathbb{D})}$, Alenka Ristić ${ }^{1}\left(\mathbb{D}\right.$, Suzana Mal $^{1,2}$, Mojca Opresnik $^{1}$ and Nataša Zabukovec Logar ${ }^{1,3} \mathbb{D}$ \\ 1 National Institute of Chemistry, Hajdrihova 19, SI-1000 Ljubljana, Slovenia; alenka.ristic@ki.si (A.R.); \\ suzana.mal@ki.si (S.M.); mojca.opresnik@ki.si (M.O.); natasa.zabukovec@ki.si (N.Z.L.) \\ 2 Jožef Stefan International Postgraduate School, Jamova Cesta 39, SI-1000 Ljubljana, Slovenia \\ 3 School of Science, University of Nova Gorica, Vipavska Cesta 13, SI-5000 Nova Gorica, Slovenia \\ * Correspondence: ciara.byrne@ki.si
}

Citation: Byrne, C.; Ristić, A.; Mal, S.; Opresnik, M.; Zabukovec Logar, N. Evaluation of ZIF-8 and ZIF-90 as Heat Storage Materials by Using Water, Methanol and Ethanol as Working Fluids. Crystals 2021, 11, 1422. https://doi.org/10.3390/ cryst11111422

Academic Editor: Volodymyr Bon

Received: 4 October 2021

Accepted: 18 November 2021

Published: 20 November 2021

Publisher's Note: MDPI stays neutral with regard to jurisdictional claims in published maps and institutional affiliations.

Copyright: (c) 2021 by the authors. Licensee MDPI, Basel, Switzerland. This article is an open access article distributed under the terms and conditions of the Creative Commons Attribution (CC BY) license (https:/ / creativecommons.org/licenses/by/ $4.0 /)$.

\begin{abstract}
The increasing demand for heating/cooling is of grave concern due to the ever-increasing population. One method that addresses this issue and uses renewable energy is Thermochemical Energy Storage (TCES), which is based on the reversible chemical reactions and/or sorption processes of gases in solids or liquids. Zeolitic imidazolate frameworks (ZIFs), composed of transition metal ions ( $\mathrm{Zn}, \mathrm{Co}$, etc.) and imidazolate linkers, have gained significant interest recently as porous adsorbents in low temperature sorption-based TES (sun/waste heat). In this study, we examined two different sodalite-type ZIF structures (ZIF-8 and ZIF-90) for their potential heat storage applications, based on the adsorption of water, methanol and ethanol as adsorbates. Both ZIF structures were analysed using PXRD, TGA, SEM and $\mathrm{N}_{2}$ physisorption while the $\%$ adsorbate uptake and desorption enthalpy was evaluated using TGA and DSC analysis, respectively. Among the studied adsorbentadsorbate pairs, ZIF-90-water showed the highest desorption enthalpy, the fastest sorption kinetics and, therefore, the best potential for use in heat storage/reallocation applications. This was due to its significantly smaller particle size and higher specific surface area, and the presence of mesoporosity as well as polar groups in ZIF-90 when compared to ZIF-8.
\end{abstract}

Keywords: ZIF-8; ZIF-90; DSC; adsorption; TCM materials

\section{Introduction}

Adsorption-based thermochemical energy storage (TCES) and reallocation rely on reversible physical adsorption and desorption processes of gases on porous solids. Adsorption is an essentially exothermic phenomenon and increased adsorption capacity of selected solid adsorbent usually leads to higher thermal energy storage capacity. At present, studies examine traditional (e.g., zeolites) and innovative (e.g., aluminophosphates and composites) sorbents for sorption thermal energy transformations [1-3]. In recent years, there has been an increased interest in metal-organic frameworks (MOFs), as they have the potential for several applications, such as catalysis and gas capture/storage [4]. MOFs are crystalline porous materials that are coordinated polymers formed from metal ion or clusters bridged by organic ligands [4,5]. One subgroup of MOFs is Zeolitic imidazolate frameworks (ZIFs) [4]. ZIFs are composed of transition metal ions (Zn, Co, etc.) and imidazolate linkers. The ZIF structure is topologically composed in a similar manner to zeolites, where the metal ion and imidazolate linker in ZIF replace the $\mathrm{Si} / \mathrm{Al}$ and $\mathrm{O}$ atoms in zeolites, respectively. ZIFs are considered to be hydrothermally stable in comparison with MOFs.

In spite of the great interest in ZIFs research, the reports on the optimization of ZIF for heat storage and reallocation applications are scarce. The majority of studies for ZIFs (and MOFs) focus on water as a working fluid [6-9]. This is due to water being cost effective and environmentally friendly [9]. However, most of the stable ZIFs are hydrophobic and, therefore, alternative working fluids have been considered. The idea of using the adsorption 
of small chain alcohols in ZIFs has already been evaluated in recent years [6,10-16]. The adsorption of methanol and ethanol in 18 MOFs (including ZIF-8) for TES applications was reported by De Lange et al. [6]. The study revealed that the use of alcohols instead of water is advantageous for the following reasons: (i) the adsorption of methanol and ethanol occurs at lower pressures; (ii) the larger pores can be used more efficiently; (iii) larger pore sizes are required to reach the desired stepwise adsorption; (iv) the effects of polar/apolar functionalities can have less impact in MOFs; (v) the energy release is lower, but the heat and mass transfer may be increased; (vi) the stability of the MOF can have less of an impact due to lower desorption temperatures; and finally (vii) cryogenic applications become a possibility [6]. Finally, the paper concludes that the use of methanol or ethanol is dependent on the evaporation temperature required for the application used [6]. HunterSellars et al. examined ZIF-8 and ZIF-67 monoliths via ligand-assisted methods for water, toluene and methanol adsorption [10]. For the monoliths, $n$-butylamine was used as the single modulating ligand while 1-methylimidazole and $n$-butylamine were used for the multiple modulating ligand monoliths. The study found that for all samples examined, water had the lowest mg/g uptake. Both unmodified ZIF-8 and ZIF-67 had a similar uptake for methanol and toluene. However, the ZIF-8 monolith using a single modulating ligand and both ZIF-67 monoliths showed a significant improvement in the uptake of methanol and toluene, favouring toluene [10]. Many studies have examined the alcohol adsorption of ZIFs for applications such as organic vapour recovery, indoor air cleaning, adsorption-based heat pumps and separation/selectivity in alcohols [6,10,15-19].

For heat storage and reallocation, numerous studies have examined MOFs/COFs $[6,9,20]$. For example, last year, Bingel et al. examined the alcohol adsorption of nanoporous materials, including MOFs, based on a meta-analysis of the literature. They concluded that while hundreds of studies based on alcohol isotherms have been conducted, these studies focused on a small group of porous materials [21]. In the same year, Li et al. studied covalent-organic frameworks (COFs) for adsorption-based heat pumps (AHPs) [9]. The study screened COFs based on grand canonical Monte Carlo simulations and machine learning. They compared the COFs with MOFs for heating, cooling and ice making. They concluded that, as adsorbents for AHPs, COFs performed better for cooling due to their weak interaction with ethanol, which favours stepwise adsorption [9]. However, ZIFs, as a subgroup, have not been studied in detail to date. Furthermore, to the best of our knowledge, there is no report on the use of short chain alcohols as working fluids for heat storage and reallocation purposes.

In order to additionally evaluate the impact of adsorbate on the energy storage performance in ZIF materials, two ZIF structures were selected and studied for the adsorption of water, methanol and ethanol. The evaluation was based on TG and DSC analyses. In general, the two main criteria for selecting ZIF structures as adsorbents are the pore entrance size and the pore/cage capacity. However, it is worth noting that the reported data for these criteria are based on static measurements and are subject to change due to a number of conditions (synthesis parameters, activation method, etc.). Additionally, it has long been known that the pore entrances are not completely rigid and may bend/relax to allow molecules into pores/cages [11,17].

The selected ZIFs were ZIF-8 and ZIF-90 (Table 1). Both ZIFs have the sample topology and pore entrance, and only have a difference of $0.4 \AA$ in their pore/cage capacities [22-24]. ZIF-8 uses the hydrophobic 2-methylimidazolate linker while ZIF90 uses the 2-carboxaldehyde imidazolate linker, which is hydrophilic [10,25]. ZIF-8 and ZIF-90 are considered the most widely researched ZIFs, with half of all articles in recent years focusing on either or both of these ZIFs [25]. As the structures have been extensively studied and are well understood, they are considered a great starting place for the evaluation of adsorption-based energy storage materials. 
Table 1. Pore entrance size $\left(\mathrm{d}_{\mathrm{a}} \mathrm{g}\right)$, the pore/cage capacity $\left(\mathrm{d}_{\mathrm{p}}{ }^{\mathrm{h}}\right)$ and topology of the ZIFs examined.

\begin{tabular}{|c|c|c|c|c|}
\hline ZIF & $\mathrm{d}_{\mathrm{a}} \mathrm{g}[\AA]$ & $\mathrm{d}_{\mathrm{p}}^{\mathrm{h}}[\AA ̊ \AA]$ & Topology & Ref. \\
\hline ZIF-8 & 3.5 & 11.6 & SOD & {$[22,23]$} \\
\hline ZIF-90 & 3.5 & 11.2 & SOD & {$[22,24]$} \\
\hline
\end{tabular}

The aim of this study was, therefore, to examine the impact that the properties of the two ZIFs had on the heat storage applications. This was completed by the synthesis ZIF-8 and ZIF-90, the examination of their structural and textural properties and the evaluation of their heat storage potential by using three different adsorbates with easily accessible sorption and calorimetric analysis.

\section{Materials and Methods}

\subsection{Materials}

2-Imidazolecarboxaldehyde (Hica, 97\%), 2-methylimidazole (mIm, 99\%), N, $\mathrm{N}$-dimethylformamide (DMF, $>99 \%)$, zinc acetate dihydrate $\left(\mathrm{ZnC}_{4} \mathrm{H}_{6} \mathrm{O}_{4} \cdot 2 \mathrm{H}_{2} \mathrm{O}, 98 \%\right)$ and zinc nitrate hexahydrate $\left(\mathrm{ZnNO}_{3} \cdot 6 \mathrm{H}_{2} \mathrm{O}, 99 \%\right)$ were purchased from Sigma Aldrich (Darmstadt, Germany). Methanol (MeOH, 99.9\%) was purchased from Honeywell Riedel-de Haën AG (Berlin, Germany). All chemicals were used without any further treatment.

\subsection{Synthesis of ZIF-8}

ZIF-8 was synthesised based on the modification of the method published by Park et al. [26]. A molar ratio of 0.25: 1.25: 0.68 was used for $\mathrm{ZnNO}_{3} \cdot 6 \mathrm{H}_{2} \mathrm{O}, \mathrm{mIm}$ and DMF, respectively. In a glass bottle, $0.265 \mathrm{~g} \mathrm{ZnNO} \cdot 6 \mathrm{H}_{2} \mathrm{O}$ and $0.067 \mathrm{~g} \mathrm{mIm}$ were weighed out, then $20 \mathrm{~mL}$ DMF was added. The solution was stirred until all the powder had fully dissolved and was then heated in an oven at $140{ }^{\circ} \mathrm{C}$ for $24 \mathrm{~h}$. The sample was filtered, washed 3 times with DMF and was dried at room temperature overnight. The sample was activated by placing in a vacuum oven at $150{ }^{\circ} \mathrm{C}$ overnight.

\subsection{Synthesis of ZIF-90}

ZIF-90 was synthesised based on the method published by Brown et al. [5]. The reaction ratio for $\mathrm{ZnC}_{4} \mathrm{H}_{6} \mathrm{O}_{4} \cdot 2 \mathrm{H}_{2} \mathrm{O}$, Hica, DMF and $\mathrm{MeOH}$ was 5 mmol.: 20 mmol.: $645 \mathrm{mmol}$ : $1.23 \mathrm{~mol}$, respectively. For the synthesis of ZIF-90, $1.920 \mathrm{~g}$ Hica $(20 \mathrm{mmol})$ and $50 \mathrm{~mL}$ DMF (645 mmol) were added in a beaker, which was heated in an oil bath at $70{ }^{\circ} \mathrm{C}$ and stirred until the solution was homogenous (solution 1). In a separate beaker, $1.485 \mathrm{~g}$ $\mathrm{ZnC}_{4} \mathrm{H}_{6} \mathrm{O}_{4} \cdot 2 \mathrm{H}_{2} \mathrm{O}(5 \mathrm{mmol})$ was added to $50 \mathrm{ml} \mathrm{MeOH}$ and stirred until the solution was completely homogenous (solution 2). Once it was fully cooled, solution 1 was slowly added to solution 2 . The resulting mixture was stirred for $30 \mathrm{~min}$ and then centrifuged at $9000 \mathrm{rpm}$ for $30 \mathrm{~min}$. The precipitate was washed with $\mathrm{MeOH}$ twice and then left to dry at room temperature overnight. In order to activate ZIF-90, the sample was soaked in $\mathrm{MeOH}$ for $7 \mathrm{~h}$, washed 3 times with fresh $\mathrm{MeOH}$ and dried in a vacuum oven overnight at $150{ }^{\circ} \mathrm{C}$.

\subsection{Characterisation}

\subsubsection{Structural Analysis}

All samples were analysed with powder X-ray Diffraction (PXRD). The PXRD patterns were produced using a PANalytical X'Pert PRO diffractometer (Malvern Panalytical, Almelo, The Netherlands), using $\mathrm{Cu} K \alpha$ radiation $(\lambda=1.5418 \AA)$. The diffraction range examined was between $2 \theta=5^{\circ}$ and $55^{\circ}$ with a step size of $0.034^{\circ}$ per $100 \mathrm{~s}$, and the examination was conducted using a fully opened 100 channel $X^{\prime}$ Celerator detector Plus. The thermal stability of each ZIF sample was determined by completing thermogravimetric analysis (TGA) with a TA Instruments Q5000 (TA Instruments, Inc., New Castle, DE, USA). TGA was repeated in the temperature range of $25-300{ }^{\circ} \mathrm{C}$ to determine the $\%$ of each adsorbate adsorbed. The analysis was performed in airflow $(25 \mathrm{~mL} / \mathrm{min})$ and was heated from 25 to $800{ }^{\circ} \mathrm{C}$ at a ramp rate of $10{ }^{\circ} \mathrm{C} / \mathrm{min}$. Brunauer- Emmett-Teller (BET) analysis 
was used to determine the specific surface area of all ZIF samples. During degassing, the samples were held at $150{ }^{\circ} \mathrm{C}$ for $10 \mathrm{~h}$. The $\mathrm{N}_{2}$ sorption isotherms were acquired at $-196^{\circ} \mathrm{C}$ on a Quantachrome AUTOSORB IQ3 (Quantachrome Instruments, Boynton Beach, FL, USA). Scanning electron microscope (SEM) images were taken using a Zeiss Supra 35 VP microscope (Carl Zeiss Microscopy, Jena, Germany) with an electron high-tension voltage of $1.00 \mathrm{kV}$ and an Aperture Size of $30.00 \mu \mathrm{m}$.

\subsubsection{Adsorption and Desorption Enthalpy Studies}

Differential scanning calorimetry (DSC) was also carried out on all samples. Prior to measurement, ZIF-8 and ZIF-90 were placed in a desiccator containing a water-saturated solution of salt $(\mathrm{NaCl}, \mathrm{rh}=75 \%)$, methanol or ethanol for 1,3 or 5 days. DSC analysis was completed on a Q2000 DSC apparatus (TA Instruments, Inc., New Castle, DE, USA) in a temperature range from 20 to $200{ }^{\circ} \mathrm{C}$ with the heating ramp of $5{ }^{\circ} \mathrm{C} / \mathrm{min}$.

\section{Results}

\subsection{Structural and Physicochemical Properties of ZIFs}

The ZIF-8 and ZIF-90 materials were synthesized using simplified literature-based procedures. Structural properties were examined using XRD, TG and nitrogen physisorption, which revealed crystalline and phase-pure products.

First, the crystalline structures of the two samples were confirmed using PXRD. These samples were compared to the simulated XRD patterns (Figure 1). XRD analysis was repeated after the activation method and showed that the crystalline structure had remained intact (Figure 1). PXRD was also used to calculate the crystallite size, which was completed using the Sherrer equation. Table 2 shows that the size of primary crystallites in ZIF-90 was 20 times higher than the size for ZIF-8.

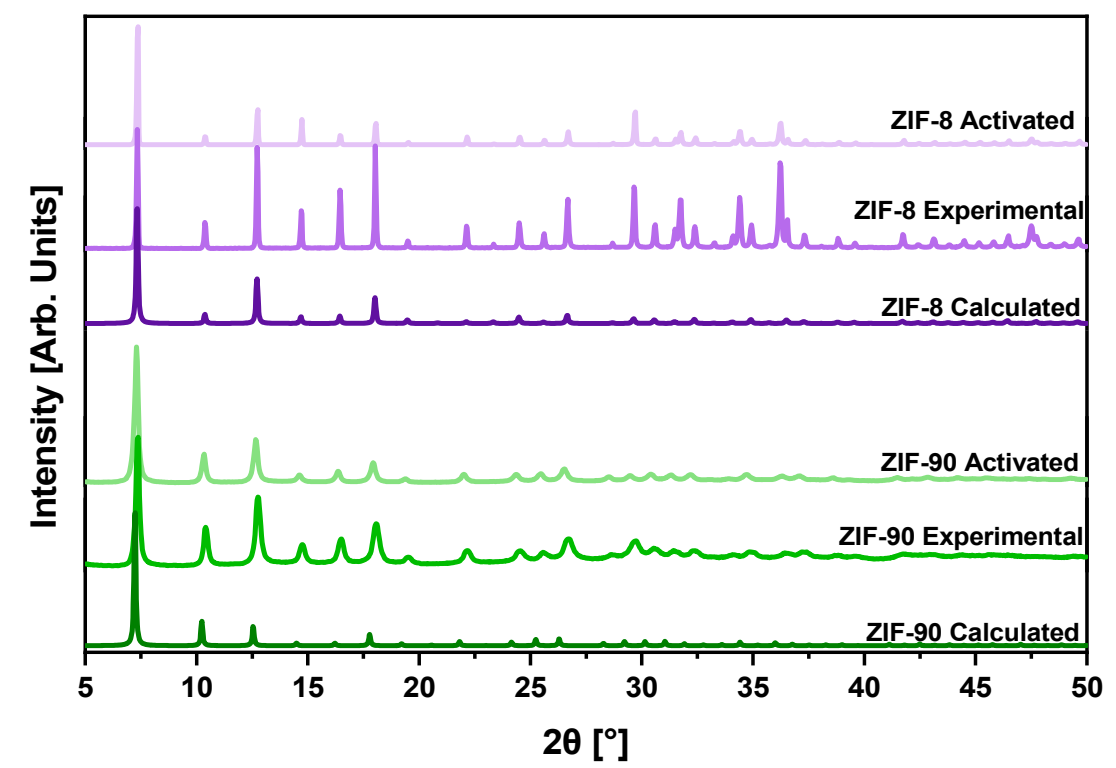

Figure 1. PXRD patterns of calculated, experimental and activated ZIF-8 (SOD) and ZIF-90 (SOD).

Table 2. Crystalline size (nm) of ZIF-8 and ZIF-90 calculated from PXRD.

\begin{tabular}{cc}
\hline ZIF & Crystalline Size (nm) \\
\hline ZIF-8 & 400 \\
ZIF-90 & 20 \\
\hline
\end{tabular}

TG analysis was performed on the as-synthesised ZIFs in order to determine at what temperature the solvent was removed from the structure and, therefore, the temperature required for the activation method. This method was repeated after the activation of the 
ZIF structures to ensure that any solvents present were removed during activation. As it can be seen in Figure 2a, there was only a slight decrease in the weight loss after the activation method was implemented, which shows that the amount of solvent in the pores of as-synthesised ZIF-8 sample was low. In contrast, ZIF-90 (Figure 2b) showed a clear and significant decrease in the peak shown at approx. $30^{\circ} \mathrm{C}$ after activation, which confirms that the majority of the substantial amount of solvent in the as-synthesized ZIF-90 was removed with activation. The weight losses at temperatures above $300^{\circ} \mathrm{C}$ were due to the gradual degradation of ZIF frameworks.
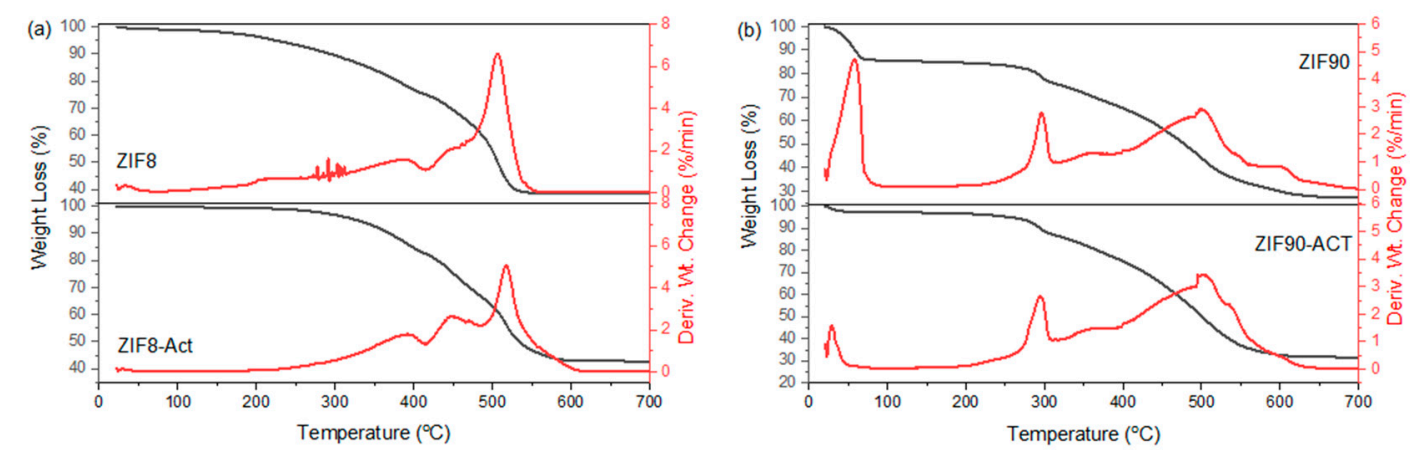

Figure 2. DTG/TG curves of as synthesized and activated (a) ZIF-8 and (b) ZIF-90.

SEM analysis was used as an additional tool to determine if the as-synthesized samples were phase-pure, confirm the morphology of the ZIFs and estimate the particle size. SEM images (Figure 3) confirmed, as with PXRD, that the as-synthesized ZIF-8 and ZIF-90 were phase-pure samples. These images also indicated that a sodalite topology was formed for both ZIFs (cubic). However, ZIF-8 (Figure 3a) was seen in individual particles while agglomerates were formed in ZIF-90 (Figure 3b). Finally, the diameter of the ZIF-8 particles was estimated to be approx. $90 \mu \mathrm{m}$. The diameter of the individual particles in the ZIF-90 agglomerate was estimated to be in range of 100 to $200 \mathrm{~nm}$, with an average of $150 \mathrm{~nm}$. This significant difference in diameter size correlates with the difference noted by the calculated crystallite sizes from PXRD. However, the primary particle size, calculated from XRPD, was even smaller (i.e., $400 \mathrm{~nm}$ for ZIF-8 and $20 \mathrm{~nm}$ for ZIF-90), which indicates that the individual particles seen in the SEM images are not single crystals, but rather, are polycrystalline.

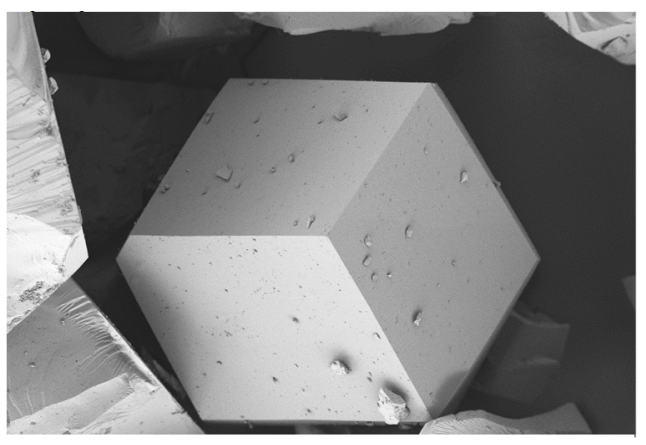

(a)

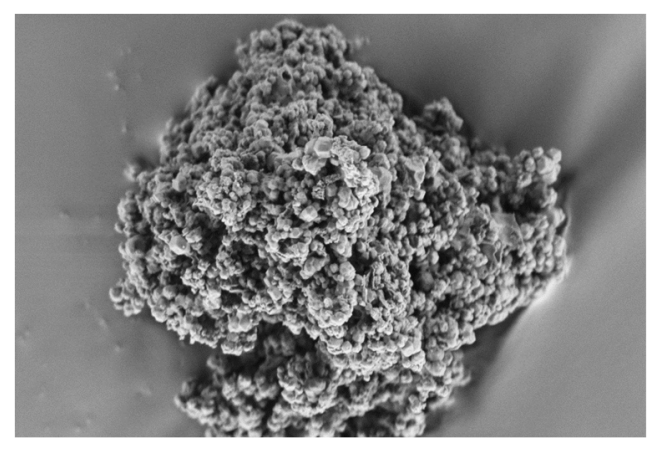

(b)

Figure 3. SEM images of as-synthesized (a) ZIF-8 and (b) ZIF-90.

Nitrogen physisorption and the BET method were used to determine the specific surface area of activated ZIF-8 and ZIF-90. Table 3 shows that the specific surface area of ZIF-90 was approx. 1.8 times higher than the specific surface area of ZIF-8. Similarly, the total pore volume of ZIF-90 was $0.571 \mathrm{~cm}^{3} / \mathrm{g}$ which was 2.3 times higher than that of ZIF-8 $\left(0.252 \mathrm{~cm}^{3} / \mathrm{g}\right)$. Figure 4 shows the nitrogen physisorption isotherms ((a) and (b)) as well as the pore size distribution ((c) and (d)). The major difference that was noted from Figure 4 
was that ZIF-8 only contained micropores while ZIF-90 was found to be made up of both micro- and mesopores. Additionally, the smaller size of the ZIF-90 particles (PXRD and SEM) compared to ZIF-8 may also have had an impact on the final specific surface area.

Table 3. BET analysis for both ZIFs showing the specific surface area $\left(\mathrm{S}_{\mathrm{BET}}\right)$ and total pore volume $\left(\mathrm{V}_{\text {total }}\right)$.

\begin{tabular}{ccc}
\hline ZIF & $\mathrm{S}_{\text {BET }}\left(\mathrm{m}^{2} / \mathbf{g}\right)$ & $\mathbf{V}_{\text {total }}\left(\mathrm{cm}^{3} / \mathbf{g}\right)$ \\
\hline ZIF-8 & 621 & 0.252 \\
ZIF-90 & 1119 & 0.571 \\
\hline
\end{tabular}
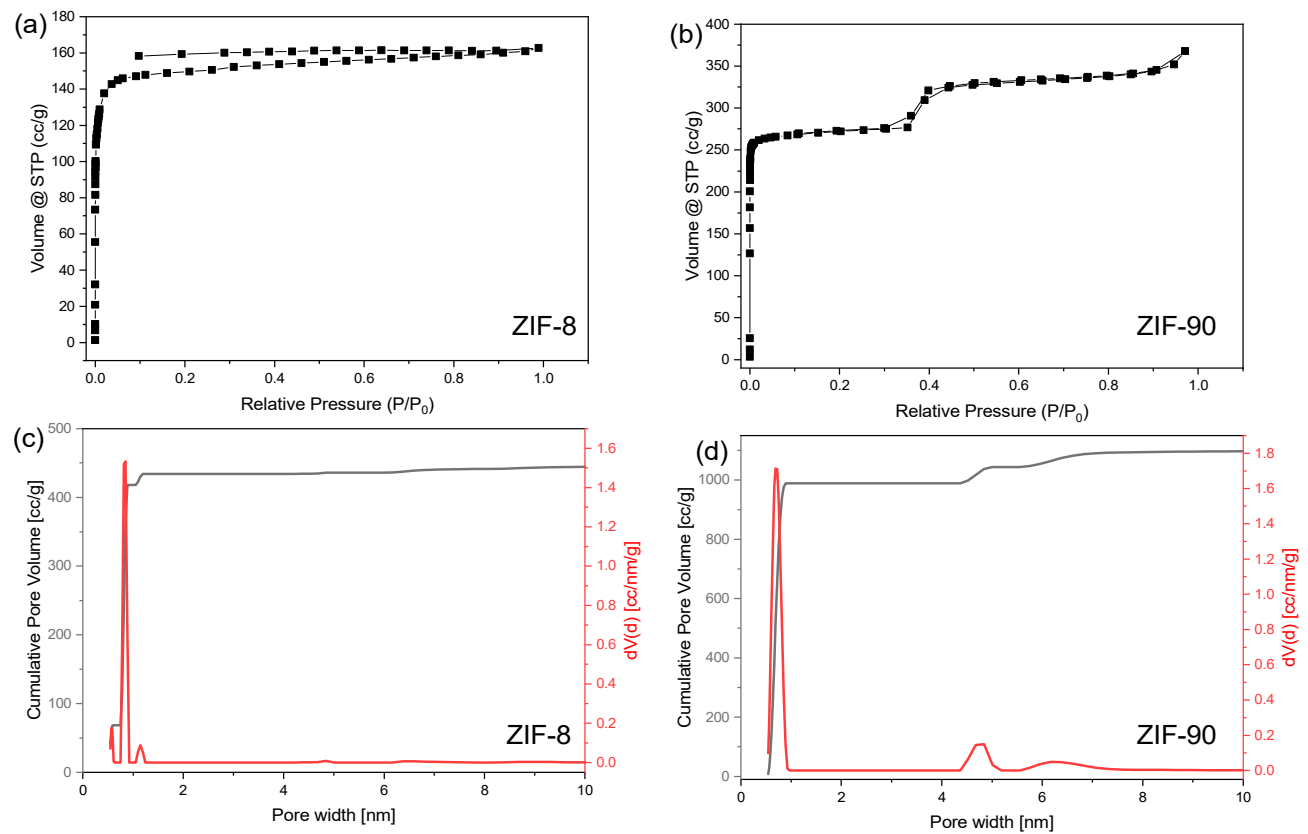

Figure 4. Nitrogen physisorption isotherms of activated (a) ZIF-8 and (b) ZIF-90 and pore size distribution of (c) ZIF-8 and (d) ZIF-90.

\section{2. $\mathrm{MeOH}, \mathrm{EtOH}$ and $\mathrm{H}_{2} \mathrm{O}$ Adsorption and DSC Analysis of ZIFs}

In order to determine the potential of using ZIF-8 and ZIF-90 as sorbents in a TES system, kinetics of adsorption and DSC analysis were performed. Each ZIF was placed in a desiccator, which contained either water, methanol or ethanol as adsorbates and left for the required number of days (1, 3 or 5 days). The $\%$ adsorbate adsorbed was examined using in-built software functions on TGA.

For ZIF-8, the adsorption of each solvent followed a different trend (Table 4). There was little variation in the \% of water adsorbed; for 1 and 3 days, it stayed constant at $1.7 \%$, while there was a decrease of $0.5 \%$ after 5 days. Ethanol showed the highest $\%$ adsorbed for all three points examined. After 1 day in the desiccator with ethanol, ZIF-8 had adsorbed $9.4 \%$ of ethanol. The \% adsorbed for 3 and 5 days stayed consistent at $12.4 \%$. Finally, methanol was the only adsorbate with an increased uptake for each time point. The \% methanol adsorbed increased from $0.9 \%$ (1 day) to $4.9 \%$ ( 5 days).

In contrast to ZIF-8, ZIF-90 had the highest \% adsorbed for all three solvents after 1 day of soaking (Table 4 ). For methanol and water, there was a decrease in $\%$ adsorbed after 3 days of soaking compared to the initial \% (1 day). Both solvents also showed a slight increase after 5 days of soaking. In contrast, ethanol showed a consistent decrease in the $\%$ adsorbed the longer the materials were kept in the desiccator. Interestingly, when comparing all solvents for 1, 3 and 5 days the adsorbate with the highest $\%$ adsorbed varied. Water soaking after 1 day showed the overall highest \% adsorbed; after 3 days of soaking, ethanol showed the highest \% adsorbed; and after 5 days of soaking, methanol 
showed the highest result. In general, the kinetics of sorption was much faster in the case of ZIF-90 as compared to the ZIF-8 material. Finally, ZIF-90 did not exhibit the same textural changes as ZIF-8 after soaking in ethanol or methanol. Namely, the ZIF-8 samples from the water desiccator remained a dry powder after 5 days of soaking. In contrast, after being placed in methanol and ethanol desiccators after 3 days, the sample became wet/paste-like. ZIF-90 did not exhibit textural changes, which were observed for ZIF-8, after being placed in the ethanol or methanol desiccators. The structural stability of both samples was, therefore, additionally checked after sorption experiments. PXRD and $\mathrm{N}_{2}$ physisorption analysis was completed after the two ZIFs had been in the three desiccators for five days. While PXRD indicated only small changes in crystallinity after soaking (Figures S5-S8), the $\mathrm{N}_{2}$ physisorption showed considerable changes in specific surface area for both samples (Table S1). For ZIF-90 samples, the values were reduced from $1119 \mathrm{~m}^{2} / \mathrm{g}$ for the activated sample to $876 \mathrm{~m}^{2} / \mathrm{g}$ and $881 \mathrm{~m}^{2} / \mathrm{g}$ for MeOH- and EtOH-treated samples, respectively. For ZIF-90 soaked in water, the decrease was the most substantial $\left(780 \mathrm{~m}^{2} / \mathrm{g}\right)$. A small decrease in specific surface area was also determined for ZIF-8 soaked in water (from 612 to $590 \mathrm{~m}^{2} / \mathrm{g}$ ). However, ZIF-8 soaked in $\mathrm{MeOH}$ and EtOH showed an increase in surface area $\left(948 \mathrm{~m}^{2} / \mathrm{g}\right.$ and $\left.919 \mathrm{~m}^{2} / \mathrm{g}\right)$. We concluded that the decrease in specific surface area and the slight lowering of intensities in XRPD were due to the partial degradation of the ZIF-90 and ZIF-8 structures during soaking in water for longer times (5 days) and, for ZIF-90, also in MeOH and EtOH. On the other hand, the soaking of ZIF-8 in alcohols contributed to the additional activation of the structure. The reduction in crystallinity and specific surface area were well aligned with the reduction in sorbent uptake when longer times of soaking ( 3 or 5 days) were employed.

Table 4. \% of solvent adsorbed for ZIF-8 and ZIF-90 after soaking for 1 day, 3 days and 5 days.

\begin{tabular}{ccccc}
\hline ZIF & Solvent & 1 Day & 3 Days & 5 Days \\
\hline \multirow{2}{*}{ ZIF-8 } & $\mathrm{MeOH}$ & $0.9 \%$ & $2.6 \%$ & $4.9 \%$ \\
& $\mathrm{EtOH}$ & $9.4 \%$ & $12.4 \%$ & $12.4 \%$ \\
& $\mathrm{H}_{2} \mathrm{O}$ & $1.7 \%$ & $1.7 \%$ & $1.2 \%$ \\
\hline \multirow{2}{*}{ ZIF-90 } & $\mathrm{MeOH}$ & $18.9 \%$ & $13.3 \%$ & $14.2 \%$ \\
& $\mathrm{EtOH}$ & $20.4 \%$ & $15.8 \%$ & $13.4 \%$ \\
\hline
\end{tabular}

Table 5 shows the desorption enthalpies or values of the energies that were needed to dry the samples of ZIF-8 and ZIF-90 after soaking in water, methanol or ethanol for 1, 3 or 5 days.

Table 5. Desorption enthalpy (J/g) for ZIF-8 and ZIF-90 after soaking for 1 day, 3 days and 5 days.

\begin{tabular}{ccccc}
\hline ZIF & Solvent & 1 Day & 3 Days & 5 Days \\
\hline \multirow{3}{*}{ ZIF-8 } & $\mathrm{MeOH}$ & 158.3 & 154.3 & 199.0 \\
& $\mathrm{EtOH}$ & 117.5 & 201.8 & 233.3 \\
& $\mathrm{H}_{2} \mathrm{O}$ & 61.9 & 52.8 & 53.2 \\
\hline \multirow{2}{*}{ ZIF-90 } & $\mathrm{MeOH}$ & 291.7 & 258.8 & 194.0 \\
& $\mathrm{EtOH}$ & 291.6 & 268.4 & 224.0 \\
& $\mathrm{H}_{2} \mathrm{O}$ & 544.3 & 304.0 & 255.1 \\
\hline
\end{tabular}

For ZIF-90, the desorption enthalpy decreased the longer it was in the desiccators containing each of the adsorbates. After being in the desiccator for 1 day, the sample in water showed the highest desorption enthalpy $(544.3 \mathrm{~J} / \mathrm{g})$ while the methanol and ethanol samples had almost the same desorption enthalpy (291.7 J/g and $291.6 \mathrm{~J} / \mathrm{g}$, respectively). As with \% adsorbed, soaking ZIF-90 in the water desiccator for 1 day showed the highest overall result. Additionally, the soaking of ZIF-90 in water also showed the highest result after 3 and 5 days (see Table 5). The desorption enthalpy decreased at a slower rate 
for ethanol when compared to methanol. After 3 days, the desorption enthalpy was $258.8 \mathrm{~J} / \mathrm{g}$ and $268.4 \mathrm{~J} / \mathrm{g}$ for the methanol and ethanol samples, respectively. This was further decreased to $194.0 \mathrm{~J} / \mathrm{g}$ (methanol) and $224.0 \mathrm{~J} / \mathrm{g}$ (ethanol).

The ZIF-8 samples from the desiccators, in comparison, showed two different trends (Table 5). The methanol and ethanol samples had an increased desorption enthalpy the longer they were left in the desiccators (though at different rates). However, the desorption enthalpy for the water sample initially decreased by $9.1 \mathrm{~J} / \mathrm{g}$ after 3 days in the desiccator and then increased slightly $(0.4 \mathrm{~J} / \mathrm{g}$ ) after 5 days (Table 5$)$. Water-adsorbed ZIF-8 showed the lowest desorption enthalpy for all three time points. Methanol-adsorbed ZIF-8 showed the best result after soaking for 1 day in the desiccators, while ethanol-adsorbed ZIF-8 showed the highest desorption enthalpy for 3 and 5 days of soaking. Finally, the sample placed in the ethanol desiccator for 5 days showed the overall highest desorption enthalpy for ZIF-8.

\section{Discussion}

Despite ZIF-8 and ZIF-90 having similar (or identical) pore entrances, pore/cage capacities and topologies (Table 1 and Figure 5), they showed significant differences in terms of crystal size, specific surface area, \% adsorbed adsorbate and associated desorption enthalpies.

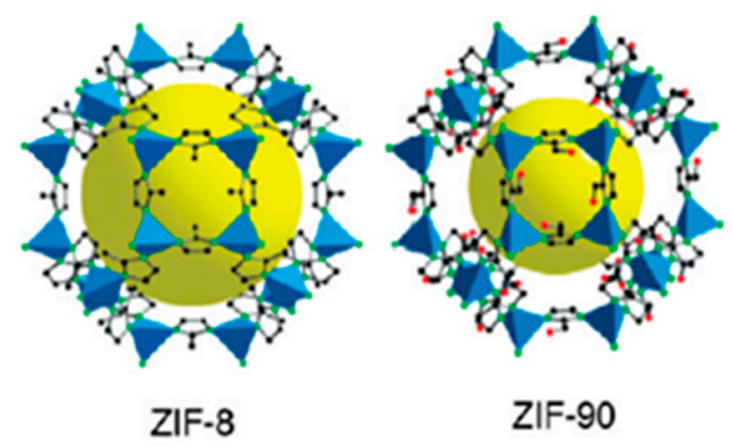

Figure 5. The sodalite topology of ZIF-8 and ZIF-90 [22]. Reprinted (adapted) with permission from Phan, A.; Doonan, C.J.; Uribe-Romo, F.J.; Knobler, C.B.; Okeeffe, M.; Yaghi, O.M. Acc. Chem. Res. 2010, 43, 58-67. Copyright 2010 American Chemical Society.

The differences in crystal sizes, specific surface areas and pore-size distributions are in accordance with the literature data. For example, the specific surface area of ZIF-90 $\left(1119 \mathrm{~m}^{2} / \mathrm{g}\right)$ is comparable with the some of the higher results reported in the literature to date, which range from 394 to $1426 \mathrm{~m}^{2} / \mathrm{g}[8,25,27-30]$. On the other hand, ZIF-8 had a specific surface area of $621 \mathrm{~m}^{2} / \mathrm{g}$. This is on the lower side, if compared to results reported in previous publications, which range from 418 to $1801 \mathrm{~m}^{2} / \mathrm{g}$, but depend greatly on the particle sizes $[8,10,16,20,31,32]$. The distribution of pore sizes in ZIF-8 (microporous) and ZIF-90 (micro-and meso-porous) are as reported in the literature $[8,25]$.

The kinetics of sorption was found to be much faster in the case of ZIF-90 if compared to ZIF-8 material. This was, as mentioned, firstly due to smaller size of crystallites and the presence of mesopores in ZIF-90, which both allow faster diffusion of adsorbate molecules. However, there are some additional reasons for the differences in the sorption behaviour of ethanol, methanol and water in ZIF-8 and ZIF-90. One reason is likely due to the hydrophobic linker used for ZIF-8, while ZIF-90 had a slightly hydrophilic linker (Figure 6). Furthermore, the hydrophobicity/hydrophilicity of the adsorbates also played a role in the energy exchange and the desorption kinetics via a possible formation of hydrogen bonds of adsorbate with the carbonyl group of ZIF-90. A pair, including hydrophobic ZIF-8 as an adsorbent and water as an adsorbate, was therefore expected to show the lowest adsorption capacity and desorption enthalpy, and our study confirmed that. On the other hand, more hydrophilic ZIF-90 and water proved to be the best performing pair also in our study. Additionally, the \% solvent adsorbed could also have been influenced by the flexibility 
of the pore entrance and structure, e.g., the pore/cage entrance flexibility of ZIF-90 was reported to be larger than for ZIF-8 in the case of methanol and ethanol, resulting in more efficient adsorption uptake in ZIF-90 [11].<smiles>O=Cc1ncc[nH]1</smiles><smiles>Cc1ncc[nH]1</smiles>

Figure 6. Showing linkers for ZIF-90 (HiCa) and ZIF-8 (mIm).

The study of adsorption capacity and associated energy exchange further showed that there is not always a direct correlation between the \% solvent adsorbed and the desorption enthalpy. It is worth noting that the texture of the ZIF-8 sample changed after soaking in methanol and ethanol, becoming a wet/paste-like, while the other samples remained powdered. The influence of textural changes could be seen for ZIF-8 soaked in ethanol for 3 and 5 days. Both time points showed a $\%$ adsorption of $12.4 \%$; however, the desorption enthalpy differed by $\sim 33 \mathrm{~J} / \mathrm{g}$. This was also seen for water-soaked ZIF-8 at 1 day and 3 days, though there was only a difference of $\sim 9 \mathrm{~J} / \mathrm{g}$.

The preference of ZIF-8 for ethanol over methanol sorption is in agreement with previous studies [11]. Examining the shape of desorption curve and the desorption peak temperature gave some further insights (see Figures S1-S4). The highest desorption enthalpy achieved was for the ZIF-90-water pair and resulted in $544.3 \mathrm{~J} / \mathrm{g}$, and $151 \mathrm{Wh} / \mathrm{kg}$. This was approx. 2.5-times lower than the results obtained for a well-known $\mathrm{AlPO}_{4}$-LTA material $(373 \mathrm{Wh} / \mathrm{kg})$, which is still one of the best performing sorption-based TES materials for low temperature applications [3]. However, one must not forget the benefits of the use of ethanol/methanol in storage applications, which were mentioned in the Introduction.

\section{Conclusions}

In this study, the evaluation of two ZIFs as adsorbents for adsorption-based energy storage, by using three different sorbates (water, methanol and ethanol), revealed a stable system suitable for potential use in TES. The ZIFs were synthesized using simplified methods. The samples were phase pure and retained crystallinity after activation. The $\%$ adsorbate adsorbed showed that the capacity varied depending on the ZIF and solvent used. The structures remained crystalline also after soaking in the three solvents for up to 5 days. ZIF-90 soaked in water for 1 day showed the highest result in \% adsorbed and desorption enthalpy. ZIF-8 had a higher infinity for ethanol adsorption compared to water and methanol; however, the maximal uptakes were a few times lower than for ZIF-90. The evaluation of energy storage potential using the DSC method proved to be a quick and effective method for assessing ZIFs in sorption-based energy storage applications.

Finally, further studies are required to fully assess the potential of using ZIFs in sorption-based energy storage applications.

Supplementary Materials: The following are available online at https:/ /www.mdpi.com/article/10 .3390/cryst11111422/s1, Figures S1-S8 and Table S1.

Author Contributions: Conceptualization, N.Z.L. and C.B.; methodology, C.B., N.Z.L. and A.R.; formal analysis, C.B., S.M. and M.O.; investigation, C.B.; data curation, C.B.; writing-original draft preparation, C.B.; writing-review and editing, N.Z.L., A.R., S.M. and M.O.; visualization, C.B.; supervision, N.Z.L.; funding acquisition, N.Z.L. All authors have read and agreed to the published version of the manuscript.

Funding: This research was funded by the Slovenian Research Agency Research program P1-0021. 
Data Availability Statement: The supporting data, including Figures S1-S8 and Table S1, are available on the journal webpage and from the authors.

Acknowledgments: The authors would like to thank Edi Kranjc for the XRD measurements.

Conflicts of Interest: The authors declare no conflict of interest.

\section{References}

1. Ristić, A.; Logar, N.Z. New composite water sorbents $\mathrm{CaCl}_{2}$-PHTS for low-temperature sorption heat storage: Determination of structural properties. Nanomaterials 2019, 9, 27. [CrossRef]

2. Henninger, S.K.; Ernst, S.J.; Gordeeva, L.; Bendix, P.; Fröhlich, D.; Grekova, A.D.; Bonaccorsi, L.; Aristov, Y.; Jaenchen, J. New materials for adsorption heat transformation and storage. Renew. Energy 2017, 110, 59-68. [CrossRef]

3. Krajnc, A.; Varlec, J.; Mazaj, M.; Ristić, A.; Logar, N.Z.; Mali, G. Superior Performance of Microporous Aluminophosphate with LTA Topology in Solar-Energy Storage and Heat Reallocation. Adv. Energy Mater. 2017, 7, 1601815. [CrossRef]

4. Ban, Y.; Peng, Y.; Zhang, Y.; Jin, H.; Jiao, W.; Guo, A.; Wang, P.; Li, Y.; Yang, W. Dual-ligand zeolitic imidazolate framework crystals and oriented films derived from metastable mono-ligand ZIF-108. Microporous Mesoporous Mater. 2016, 219, 190-198. [CrossRef]

5. Brown, A.J.; Johnson, J.R.; Lydon, M.E.; Koros, W.J.; Jones, C.W.; Nair, S. Continuous polycrystalline zeolitic imidazolate framework-90 membranes on polymeric hollow fibers. Angew. Chemie Int. Ed. 2012, 51, 10615-10618. [CrossRef]

6. De Lange, M.F.; Van Velzen, B.L.; Ottevanger, C.P.; Verouden, K.J.F.M.; Lin, L.C.; Vlugt, T.J.H.; Gascon, J.; Kapteijn, F. MetalOrganic Frameworks in Adsorption-Driven Heat Pumps: The Potential of Alcohols as Working Fluids. Langmuir 2015, 31, 12783-12796. [CrossRef]

7. Lalonde, M.B.; Mondloch, J.E.; Deria, P.; Sarjeant, A.A.; Al-Juaid, S.S.; Osman, O.I.; Farha, O.K.; Hupp, J.T. Selective SolventAssisted Linker Exchange (SALE) in a Series of Zeolitic Imidazolate Frameworks. Inorg. Chem. 2015, 54, 7142-7144. [CrossRef]

8. Gao, M.; Wang, J.; Rong, Z.; Shi, Q.; Dong, J. A combined experimental-computational investigation on water adsorption in various ZIFs with the SOD and RHO topologies. RSC Adv. 2018, 8, 39627-39634. [CrossRef]

9. Li, W.; Xia, X.; Li, S. Screening of Covalent-Organic Frameworks for Adsorption Heat Pumps. ACS Appl. Mater. Interfaces 2020, 12, 3265-3273. [CrossRef]

10. Hunter-Sellars, E.; Saenz-Cavazos, P.A.; Houghton, A.R.; McIntyre, S.R.; Parkin, I.P.; Williams, D.R. Sol-Gel Synthesis of HighDensity Zeolitic Imidazolate Framework Monoliths via Ligand Assisted Methods: Exceptional Porosity, Hydrophobicity, and Applications in Vapor Adsorption. Adv. Funct. Mater. 2021, 31, 2008357-2008367. [CrossRef]

11. Gee, J.A.; Chung, J.; Nair, S.; Sholl, D.S. Adsorption and diffusion of small alcohols in zeolitic imidazolate frameworks ZIF-8 and ZIF-90. J. Phys. Chem. C 2013, 117, 3169-3176. [CrossRef]

12. Gao, C.; Shi, Q.; Dong, J. Adsorptive separation performance of 1-butanol onto typical hydrophobic zeolitic imidazolate frameworks (ZIFs). CrystEngComm 2016, 18, 3842-3849. [CrossRef]

13. Eum, K.; Jayachandrababu, K.C.; Rashidi, F.; Zhang, K.; Leisen, J.; Graham, S.; Lively, R.P.; Chance, R.R.; Sholl, D.S.; Jones, C.W.; et al. Highly tunable molecular sieving and adsorption properties of mixed-linker zeolitic imidazolate frameworks. J. Am. Chem. Soc. 2015, 137, 4191-4197. [CrossRef] [PubMed]

14. Wei, W.; Huang, J.; Li, W.; Peng, H.; Li, S. Impacts of Ethanol and Water Adsorptions on Thermal Conductivity of ZIF-8. J. Phys. Chem. C 2019, 123, 27369-27374. [CrossRef]

15. Tang, Y.; Dubbeldam, D.; Guo, X.; Rothenberg, G.; Tanase, S. Efficient Separation of Ethanol-Methanol and Ethanol-Water Mixtures Using ZIF-8 Supported on a Hierarchical Porous Mixed-Oxide Substrate. ACS Appl. Mater. Interfaces 2019, 11, 21126-21136. [CrossRef]

16. Zhang, K.; Lively, R.P.; Dose, M.E.; Brown, A.J.; Zhang, C.; Chung, J.; Nair, S.; Koros, W.J.; Chance, R.R. Alcohol and water adsorption in zeolitic imidazolate frameworks. Chem. Commun. 2013, 49, 3245-3247. [CrossRef]

17. Du, Y.; Mao, K.; Wooler, B.; Sharma, A.K.; Colmyer, D.; Nines, M.; Weston, S.C. Insights into the Flexibility of ZIF-7 and Its Structural Impact in Alcohol Adsorption. J. Phys. Chem. C 2017, 121, 28090-28095. [CrossRef]

18. Virdis, T.; Danilov, V.; Baron, G.V.; Denayer, J.F.M. Nonideality in the Adsorption of Ethanol/Ethyl Acetate/Water Mixtures on ZIF-8 Metal Organic Framework. Ind. Eng. Chem. Res. 2018, 57, 7040-7047. [CrossRef]

19. Yin, H.; Lau, C.Y.; Rozowski, M.; Howard, C.; Xu, Y.; Lai, T.; Dose, M.E.; Lively, R.P.; Lind, M.L. Free-standing ZIF-71/PDMS nanocomposite membranes for the recovery of ethanol and 1-butanol from water through pervaporation. J. Memb. Sci. 2017, 529, 286-292. [CrossRef]

20. Xia, X.; Liu, Z.; Li, S. Adsorption characteristics and cooling/heating performance of COF-5. Appl. Therm. Eng. 2020, 176, 115442-115450. [CrossRef]

21. Bingel, L.W.; Chen, A.; Agrawal, M.; Sholl, D.S. Experimentally Verified Alcohol Adsorption Isotherms in Nanoporous Materials from Literature Meta-Analysis. J. Chem. Eng. Data 2020, 65, 4970-4979. [CrossRef]

22. Phan, A.; Doonan, C.J.; Uribe-romo, F.J.; Knobler, C.B.; Keeffe, M.O.; Yaghi, O.M. Synthesis, Structure, and Carbon Dioxide Capture Properties of Zeolitic Imidazolate Frameworks. Acc. Chem. Res. 2010, 43, 58-67. [CrossRef] [PubMed]

23. Huang, X.; Zhang, J.; Chen, X. [Zn(bim $\left.)_{2}\right] \cdot\left(\mathrm{H}_{2} \mathrm{O}\right) 1.67$ : A metal-organic open-framework with sodalite topology. Chin. Sci. Bull. 2003, 48, 1531-1534. [CrossRef] 
24. Morris, W.; Doonan, C.J.; Furukawa, H.; Banerjee, R.; Yaghi, O.M. Crystals as molecules: Postsynthesis covalent functionalization of zeolitic imidazolate frameworks. J. Am. Chem. Soc. 2008, 130, 12626-12627. [CrossRef] [PubMed]

25. Škrjanc, A.; Byrne, C.; Zabukovec Logar, N. Green Solvents as an Alternative to DMF in ZIF-90 Synthesis. Molecules 2021, $26,1573$. [CrossRef] [PubMed]

26. Park, K.S.; Ni, Z.; Côté, A.P.; Choi, J.Y.; Huang, R.; Uribe-Romo, F.J.; Chae, H.K.; O’Keeffe, M.; Yaghi, O.M. Exceptional chemical and thermal stability of zeolitic imidazolate frameworks. Proc. Natl. Acad. Sci. USA 2006, 103, 10186-10191. [CrossRef]

27. Ghahramaninezhad, M.; Mohajer, F.; Niknam Shahrak, M. Improved $\mathrm{CO}_{2}$ capture performances of ZIF-90 through sequential reduction and lithiation reactions to form a hard/hard structure. Front. Chem. Sci. Eng. 2020, 14, 425-435. [CrossRef]

28. Zhang, Q.; Luo, S.; Weidman, J.; Guo, R. Surface modification of ZIF-90 with triptycene for enhanced interfacial interaction in mixed-matrix membranes for gas separation. J. Polym. Sci. 2020, 58, 2675-2687. [CrossRef]

29. Shieh, F.K.; Wang, S.C.; Leo, S.Y.; Wu, K.C.W. Water-based synthesis of zeolitic imidazolate framework-90 (ZIF-90) with a controllable particle size. Chem. A Eur. J. 2013, 19, 11139-11142. [CrossRef]

30. Zhang, H.; Duan, C.; Li, F.; Yan, X.; Xi, H. Green and rapid synthesis of hierarchical porous zeolitic imidazolate frameworks for enhanced $\mathrm{CO}_{2}$ capture. Inorganica Chim. Acta 2018, 482, 358-363. [CrossRef]

31. Thompson, J.A.; Chapman, K.W.; Koros, W.J.; Jones, C.W.; Nair, S. Sonication-induced Ostwald ripening of ZIF-8 nanoparticles and formation of ZIF-8/polymer composite membranes. Microporous Mesoporous Mater. 2012, 158, 292-299. [CrossRef]

32. Nordin, N.A.H.M.; Ismail, A.F.; Mustafa, A.; Murali, R.S.; Matsuura, T. The impact of ZIF-8 particle size and heat treatment on $\mathrm{CO}_{2} / \mathrm{CH}_{4}$ separation using asymmetric mixed matrix membrane. RSC Adv. 2014, 4, 52530-52541. [CrossRef] 